A Wave Finite Element Analysis of the Passive Cochlea

Stephen J. Elliott

Institute of Sound and Vibration Research, University of Southampton, Highfield Campus, Southampton SO17 1BJ United Kingdom

\title{
Guangjian Ni
}

Institute of Sound and Vibration Research, University of Southampton, Highfield Campus, Southampton SO17 1BJ United Kingdom

Brian R. Mace

Department of Mechanical Engineering, University of Auckland, City Campus

Auckland 1142 New Zealand

\section{Ben Lineton}

Institute of Sound and Vibration Research, University of Southampton, Highfield Campus, Southampton SO17 1BJ United Kingdom 


\begin{abstract}
Current models of the cochlea can be characterised as being either based on the assumed propagation of a single slow wave, which provides good insight, or involve the solution of a numerical model, such as in the finite element method, which allows the incorporation of more detailed anatomical features. In this paper it is shown how the wave finite element method can be used to decompose the results of a finite element calculation in terms of wave components, which allows the insight of the wave approach to be brought to bear on more complicated numerical models. In order to illustrate the method, a simple box model is considered, of a passive, locally reacting, basilar membrane interacting via three-dimensional fluid coupling. An analytic formulation of the dispersion equation is used initially, to illustrate the types of wave one would expect in such a model. The wave finite element is then used to calculate the wavenumbers of all the waves in the finite element model. It is shown that only a single wave type dominates the response until this peaks at the best place in the cochlea, where an evanescent, higher order fluid wave can make a significant contribution.
\end{abstract}




\section{Introduction}

Most descriptions of the mechanical response of the cochlea involve the forward propagation of a single, "slow", wave (Zwislocki, 1950; Zweig et al., 1976; de Boer, 1996). This wave is generated by the interaction between the inertia of the fluid in the chambers of the cochlea and the stiffness of the basilar membrane, BM, and can be reproduced with simple onedimensional box models (de Boer, 1996). At low sound pressure levels the amplitude of this wave is amplified by a number of active processes within the organ of Corti, but the basic description of slow wave propagation is valid even when the cochlea is passive, as it is for high sound pressure levels. Since the properties of the cochlea, particularly the BM stiffness, vary along its length, the properties of this slow wave are position-dependent when excited at a given driving frequency. These properties can be characterised at each position along the cochlea by a complex wavenumber, whose real part determines the wave speed and whose imaginary part determines the spatial attenuation of the wave. If the wavenumber distribution along the cochlea can be calculated from a model, or inferred using an inverse method from measurements (Shera, 2007), the mechanical response of the cochlea can then be calculated using the Wentzel-Kramers-Brillouin, WKB, method (Zweig et al., 1976).

The WKB method has a number of inherent assumptions, however, such as that the wave is only travelling in one direction. This implies that no backward travelling wave is generated by the normal hearing function of the cochlea, even though such waves are believed to be responsible for other phenomena, such as otoacoustic emissions for example. Another assumption is that the wavenumber does not vary too rapidly with position, as compared with the wavelength (de Boer, 1996), although this assumption does not appears to limit the applicability of the WKB method in cochlea modelling as much as one would expect (de Boer and MacKay, 1980). Another inherent assumption is that only a single type of wave is responsible for the mechanical response of the cochlea. 
The discussion of multiple wave types in the cochlea is not new. Steele and Taber (1979a) and Taber and Steele (1981), for example, used a Lagrangian approach to derive a dispersion relation, corresponding to the eikonal equation in the WKB method, for waves in the passive cochlea. For 2D and 3D fluid coupling, the effective height of the fluid chamber is a transcendental function of the wavenumber and this leads to an infinite number of wavenumbers that satisfy the dispersion equation, and hence multiple wave types. These authors note that the most difficult part of their numerical computation is the extraction of "the necessary root" of this equation that corresponds to the travelling wave solution that they are seeking. Their WKB solutions are then constructed from this single wave type. Similarly de Boer and Viergever (1982) derive dispersion equations for 2D and 3D fluid coupling, noting that they have multiple roots and describe methods by which a single wavenumber may be selected corresponding to "the correct solution".

Both these authors, and Steele and Taber (1979a), note a difference between the WKB solution for the distribution of the complex BM motion along the cochlea and the full numerical solution, just apical of peak response. de Boer and Viergever (1982) suggest that this is because the "wrong" solution of the dispersion equation has been chosen. Chadwick et al. (1996) describe an analytic model of a slice of the cochlea having subpartitions and four fluid chambers. They also derive a dispersion equation, which in their case is quartic and so yields four roots. It is noted that some roots represent non-propagating waves and a single wavenumber is chosen for a given model to represent the propagating wave in their asymptotic formulation. Steele (1999) also describes how multi-chamber models give rise to multiple modes. Cai et al. $(2003,2004)$ discuss how a more detailed numerical model of slices of the cochlea can be used to describe wave propagation. In this case a finite element (FE) model of the 2D cross-section was constructed and used to calculate multiple values of the wavenumber, from which the one with the least-negative imaginary part is selected for a 
WKB solution over the length of the cochlea. In each of these models it has been assumed that a single wave type dominates the overall response of the cochlea. Watts (2000) returned to the observed difference between the numerical and WKB solutions beyond the peak and discussed how it could be explained with a second wave mode, which is necessary to satisfy the fluid coupling equation. There has also been recent interest in mode conversion in a two chamber model of the cochlea (Lamb and Chadwick, 2011).

The purpose of this paper is to introduce a method of analysing a detailed numerical model of the cochlea into its constitutive wave components, using the wave finite element (WFE) method (Mace et al., 2005). Similar methods have previously been used to analyse wave propagation in uniform engineering structures, such as railway lines (Thompson, 1993) and tyres (Waki et al., 2009a). The wave finite element method is used here to calculate the position-dependent characteristics of the waves that are able to propagate through individual sections of a cochlear model. An advantage of this method over that described by Cai and Chadwick (2003), for example, is that these sections can have a finite length and hence internal structure, although this aspect of the method is not exploited here. The main difference between this and previous work, however, is that we are not seeking to select a single wave type, in order to improve the computational efficiency of the model by adopting a WKB approach, but are using the calculated properties of these different wave types to decompose the results of a full finite element analysis into individual wave components. The main aim of the method is thus to test the assumption that only a single wave propagates along the cochlea and quantify the extent to which this assumption is valid. In this initial paper the wave finite element method is described and its use in decomposing the results of a full finite element model into wave components is discussed. In order to compare the results with the previous models described above, only a simple passive cochlear model is considered. We show that the results of the full finite element model can be accurately 
represented over most of the cochlear length by the forward propagation of a single slow wave. The response beyond the peak can involve multiple wave types, however, as predicted by Watts (2000), which are identified here as higher order acoustic waves in the fluid coupling. Although the method is illustrated only for the passive model, whose wave behaviour is reasonably well understood, it has general applicability to more complicated numerical models, provided they are linear. The importance of the wave finite element method is thus its generality. The approach can be used on more detailed finite element models of the cochlea, in order to systematically investigate the importance, for example, of more complicated fluid coupling, longitudinal coupling along elements of the organ of Corti or electrical coupling between the hair cells.

An analytic model of wave propagation in the cochlea is briefly reviewed in Sec. II, since this provides an idea of the properties of the additional wave types that we would expect to encounter in our model of the passive cochlea. In Sec. III, the response of a passive 3D box model of the cochlea is predicted from a full finite element model, in order to describe the fluid-structural coupling and issues of meshing. The wave finite element method is introduced in Sec. III and used to analyse the types of wave that could propagate in the cochlea. This method is then used in Sec. IV to decompose the results of the full finite element model into individual wave components.

\section{Wavenumber Analysis}

Before using the wave finite element method to calculate the properties of the waves that can propagate in the cochlea, we first review an analytic approach to obtain their wavenumber distributions. This will help in the interpretation of the numerical results. We assume a box model for the cochlea, as shown in Fig. 1, in which the two symmetrical fluid chambers are 
separated by a rigid partition that includes the flexible BM on one side. To calculate the fluid coupling, the BM is assumed to vibrate as a single mode, so that its displacement is

$$
w(x, y)=\frac{v_{\mathrm{BM}}(x)}{i \omega} \sin \frac{\pi y}{B},
$$

where $x$ and $y$ are the longitudinal and radial variables, $B$ is the $\mathrm{BM}$ width and $v_{\mathrm{BM}}(x)$ is the complex modal velocity of the BM (Elliott et al., 2011).

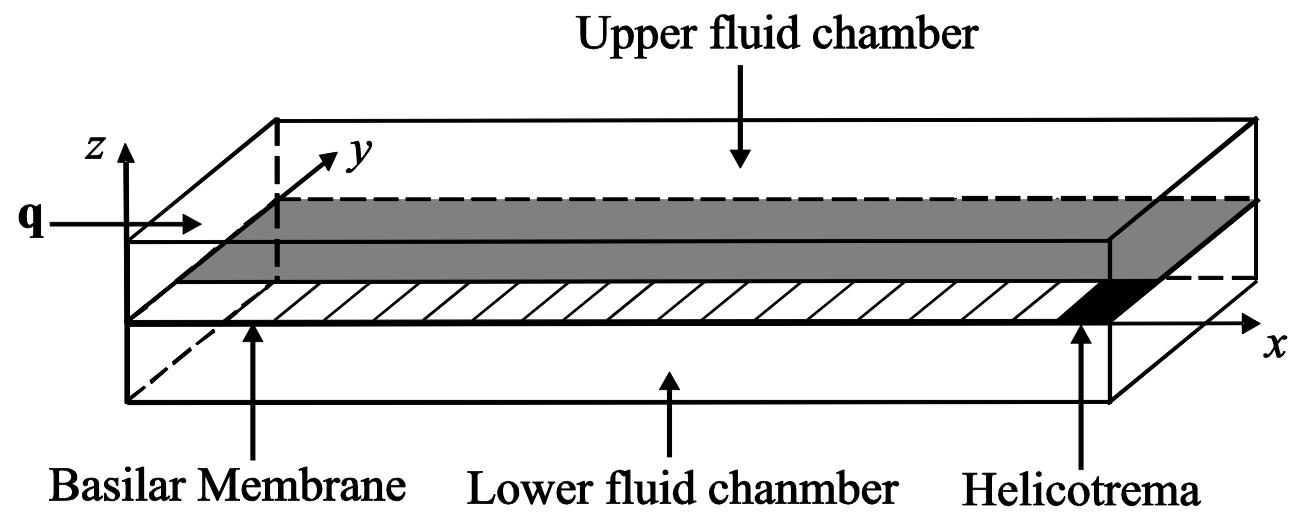

Fig. 1 The box model of the cochlea in which two fluid chambers, connected at the helicotrema, are separated by a partition that includes the flexible basilar membrane (BM).

We also define the modal pressure acting on the BM as

$$
p(x)=\frac{1}{B} \int_{0}^{B} p_{\mathrm{d}}(x, y) \sin \frac{\pi y}{B} d y,
$$

where $p_{\mathrm{d}}(x, y)$ is the distribution of complex pressure difference acting on the BM. By assuming that a wave with complex wavenumber $k$ propagates in the cochlea, the modal pressure for this wavenumber can be related to the corresponding modal BM velocity by a wavenumber-dependent fluid coupling impedance (Steele and Taber, 1979; Elliott et al., 2011)

$$
\frac{P(k)}{V(k)}=Z_{\mathrm{FC}}(k) .
$$

If we assume that the $\mathrm{BM}$ is locally reacting, so its velocity response at position $x$ only depends on the pressure at this position, we can also write the modal BM velocity as 


$$
v(x)=-\frac{p(x)}{Z_{\mathrm{BM}}(x)}
$$

where the negative sign indicating that positive pressure difference generates negative BM velocity, and $Z_{\mathrm{BM}}(x)$ is the local impedance of the BM. Although $Z_{\mathrm{BM}}$ must vary with position, at a given frequency, for the normal functioning of the cochlea, it is assumed that this variation is slow compared with the wavelength, so that in the wavenumber domain it can be taken to be constant. By combining Eq. (3) and (4) the dispersion equation for the wave is then obtained as

$$
Z_{\mathrm{FC}}(k)+Z_{\mathrm{BM}}=0,
$$

which is a special case of the formulation of Chadwick and Dimitriadis (1996) for two fluid chambers.

If only one-dimensional fluid coupling is assumed, then the fluid coupling impedance takes simple form (Steele and Taber, 1979; de Boer, 1996; Elliott et al., 2011)

$$
Z_{\mathrm{FC}}=\frac{2 i \omega \rho}{k^{2} h},
$$

where $h$ is the effective height of the fluid chamber, given for the geometry in Fig. 1 by $\pi^{2} W H / 8 B$, where $W$ and $H$ are the physical width and height of the fluid chamber and $B$ is again the BM width. The dispersion equation, Eq. (5), can be solved in this case to give an explicit solution for the wavenumber as

$$
k(x)= \pm \sqrt{\frac{-2 i \omega \rho}{h Z_{\mathrm{BM}}(x)}},
$$

where the slow dependence of $Z_{\mathrm{BM}}$, and hence $k$, on $x$ has now been re-introduced, and the two roots correspond to forward and backward travelling waves.

The full three-dimensional form of the fluid coupling impedance takes the form of an infinite series of hyperbolic functions (Steele and Taber, 1979; Elliott et al., 2011), and so the 
dispersion equation in this case has an infinite number of solutions (Taber and Steele, 1981). Insight into the form of these solutions can, however, be obtained by taking a polynomial approximation to this infinite series, the fourth order version of which can be written as (de Boer, 1998)

$$
Z_{\mathrm{FC}}(k)=\frac{2 i \omega \rho}{k^{2} h}\left(\frac{1+a k^{2}}{1+b k^{2}}\right),
$$

where $a$ and $b$ are fitted parameters having the dimensions of length squared. The dispersion equation then takes the form

$$
b h Z_{\mathrm{BM}} k^{4}+\left(h Z_{\mathrm{BM}}+2 a i \omega \rho\right) k^{2}+2 i \omega \rho=0,
$$

which has four roots, corresponding to two separate types of waves propagating in each direction.

Figure 2 shows the spatial distribution of the real and imaginary parts of the wavenumbers for the two forward going waves at $1 \mathrm{kHz}$, obtained by solving Eq. (9). We have assumed that the passive BM dynamics can be represented by the single degree of freedom model

$$
Z_{\mathrm{BM}}(x)=i \omega m(x)+\frac{s(x)}{i \omega}+r(x)
$$

where $m(x), s(x)$ and $r(x)$ are the mass, stiffness and damping, per unit area, at a given position along the cochlea. For purposes of illustration we have assumed that the fluid chambers have the same size all along the cochlea and that the BM mass per unit area is also constant, with a value of $m_{0}$. This is taken as $0.03 \mathrm{~kg} / \mathrm{m}^{2}$ for the $3 \mathrm{D}$ model, corresponding to a physically reasonable value of $30 \mu \mathrm{m}$ for the mean BM and organ of Corti thickness. An additional added mass due to the fluid loading is also present in this case, which can be calculated by taking the limiting case of Eq. (8), as $k$ tends to zero (Elliott et al., 2011). This is equal to $2 \rho(a-b) / h$, or about $0.27 \mathrm{~kg} / \mathrm{m}^{2}$ for the parameter values used here, so that in the 1D case, where this additional mass must be included in the effective BM mass, $m_{0}$ is taken as $0.3 \mathrm{~kg} / \mathrm{m}^{2}$. 

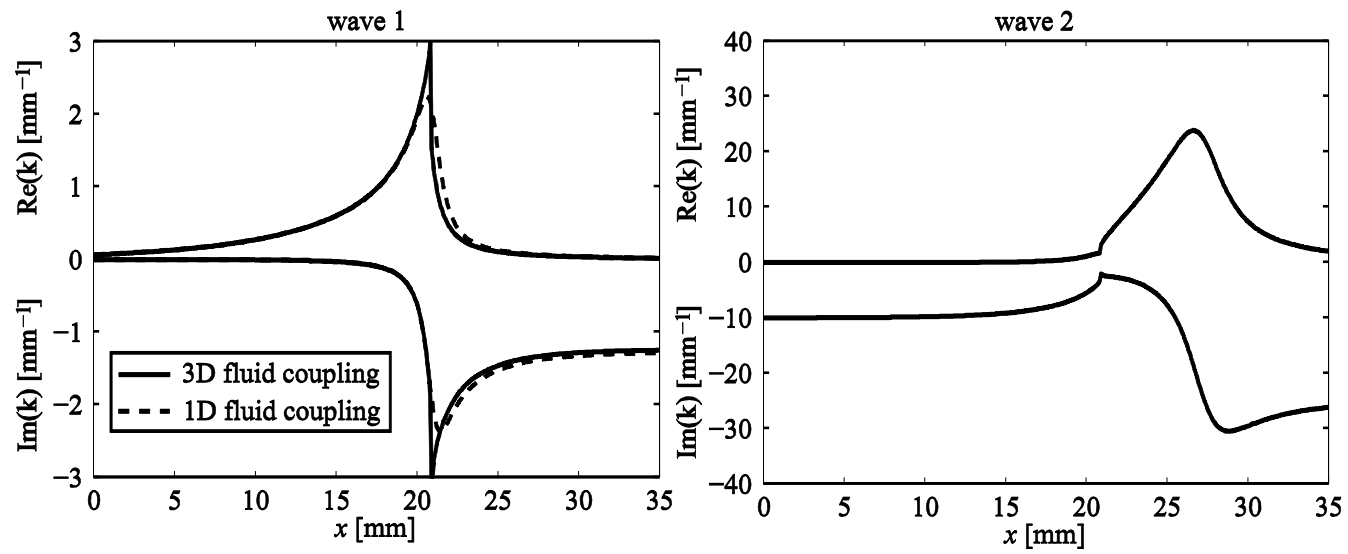

Fig. 2 The spatial distribution of the real and imaginary parts of the wavenumbers of the two forward going waves, wave 1 above and wave 2 below, when calculated from a model with an approximation to 3D fluid coupling at $1 \mathrm{kHz}$. The wavenumber of the first, slow, wave calculated using only 1D fluid coupling is shown as dashed.

The local natural frequency of the BM, $\omega_{\mathrm{n}}(x)$, equal to $\sqrt{s(x) / m(x)}$, is assumed to vary exponentially along the cochlea, so that

$$
\omega_{\mathrm{n}}(x)=\omega_{\mathrm{B}} e^{-x / l},
$$

where $\omega_{\mathrm{B}}$ is the natural frequency at the base and $l$ is the length scale for the natural frequency variation. The stiffness distribution along the cochlea is thus given by

$$
s(x)=\omega_{\mathrm{B}}^{2} m_{0} e^{-2 x / l} .
$$

Also, by assuming a constant damping ratio for the $\mathrm{BM}, \zeta_{0}$, the damping distribution is given by

$$
r(x)=2 \zeta_{0} m_{0} \omega_{\mathrm{B}} e^{-x / l}
$$

The values of all the parameters used are listed in Table 1.

The wavenumber distribution of the first wave in Fig. 2, denoted wave 1, is similar to that calculated using Eq. (7) from the 1D fluid coupling analysis, which is shown, as the dashed line, in Fig. 2. This corresponds to the conventional slow cochlear wave. For $x$ less than about $20 \mathrm{~mm}$, the real part of the wavenumber gradually increases along the cochlea, showing that 
the wave is slowing down, and the imaginary part of the wavenumber is small, indicating that it is not significantly attenuated. Beyond this position the imaginary part of the wavenumber is negative, with an almost constant value of order $-1 \mathrm{~mm}^{-1}$, indicating that the wave is decaying exponentially with an exponential decay length of about $1 \mathrm{~mm}$. This is in good agreement with the result obtained from Eq. (7) if the high frequency limit for $Z_{\mathrm{BM}}(x)$ is taken in Eq. (10), so that it is equal to $i \omega m_{0}$.

Table 1 Assumed parameters of the passive cochlear model.

\begin{tabular}{|c|c|c|}
\hline Parameter & Symbol & Assumed value \\
\hline Total length & $L$ & $35 \mathrm{~mm}$ \\
\hline Width of fluid chamber & $W$ & $1 \mathrm{~mm}$ \\
\hline Height of fluid chamber & $H$ & $1 \mathrm{~mm}$ \\
\hline Width of the BM & $B$ & $0.3 \mathrm{~mm}$ \\
\hline Natural frequency length scale & $l$ & $7 \mathrm{~mm}$ \\
\hline Effective height of fluid chamber & $h$ & $4.1 \mathrm{~mm}$ \\
\hline Natural frequency at base & $\omega_{\mathrm{B}}$ & $2 \pi \times 20 \mathrm{kHz}$ \\
\hline 1D BM mass & $m_{0(1 \mathrm{D})}$ & $0.3 \mathrm{~kg} \mathrm{~m}^{-2}$ \\
\hline 3D BM mass & $m_{0(3 \mathrm{D})}$ & $0.03 \mathrm{~kg} \mathrm{~m}^{-2}$ \\
\hline BM damping ratio & $\zeta_{0}$ & 0.1 \\
\hline \multirow[t]{2}{*}{ Parameter in $3 \mathrm{D} \mathrm{Z} \mathrm{Z}_{\mathrm{FC}}$} & $a$ & $5.5 \times 10^{-7} \mathrm{~m}^{2}$ \\
\hline & $b$ & $1 \times 10^{-8} \mathrm{~m}^{2}$ \\
\hline
\end{tabular}

The second wave, shown as wave 2 in Fig. 2, has a significant imaginary component even at the base, with a value of about $(0.1 \mathrm{~mm})^{-1}$. Using the definition of $Z_{\mathrm{FC}}(k)$ in Eq. (3), and its assumed form in Eq. (8), we can derive an equation relating the pressure and velocities in the wavenumber domain as

$$
P(k) k^{2} h\left(1+b k^{2}\right)=2 i \omega \rho\left(1+a k^{2}\right) V(k) .
$$

If the $\mathrm{BM}$ were rigid, so that $V(k)$ was equal to zero and the wave propagated in the fluid only, then $k$ could either be zero, corresponding to a fast wave of infinite speed, or equal to $\pm i \sqrt{1 / b}$. The later condition corresponds to an evanescent wave, with a decay length of 0.1 
$\mathrm{mm}$, for the value of $b$ assumed here, which agrees well with the observed properties of the second wave near the base. If higher order approximations than Eq. (8) are used for the fluid coupling impedance, further evanescent higher-order fluid waves are generated.

The interaction between the BM and the fluid becomes more significant for this wave further along the cochlea, but for $x$ less than about $20 \mathrm{~mm}$, the two waves can be identified as a coupled slow wave, as predicted by the 1D fluid coupling case, and an evanescent fluid wave, which physically corresponds to a cut off higher-order acoustic mode in the fluid chamber (Elliott et al., 2011).

\section{The Finite Element Model}

\section{a) Full Finite Element}

A finite element version of the cochlear box model is obtained by dividing its length into 512 elements, in the $x$ direction, and each fluid chamber into an $8 \times 4$ grid of hexahedral elements, in the $y \times z$ directions. Using symmetry it is only necessary to include a single fluid chamber in the numerical model. The BM within each of the 512 elements is modelled as a 4 element thin plate strip, with no longitudinal coupling between each plate. Each plate thus vibrates independently in the absence of the fluid and provides a locally reacting model of the BM, consistent with the model in Sec. II. The displacement of the all plate elements is represented by the vector $\mathbf{w}$, so that their undamped dynamics can be written in the matrix form

$$
\mathbf{M} \ddot{\mathbf{w}}+\mathbf{K w}=\mathbf{S p}
$$

where $\mathbf{M}$ and $\mathbf{K}$ are the mass and stiffness matrices for the plate, $\ddot{\mathbf{w}}$ represents $\partial^{2} \mathbf{w} / \partial t^{2}$ and $\mathbf{p}$ is the vector of pressures in the elements of the fluid chamber, which drive the plate via the coupling matrix $\mathbf{S}$.

The dynamic response of the fluid can also be represented in finite element form (Fahy and Gardonio, 2007) as 


$$
\mathbf{Q} \ddot{\mathbf{p}}+\mathbf{H p}=-\rho_{f} \mathbf{R} \ddot{\mathbf{w}}+\mathbf{q}
$$

where $\mathbf{Q}$ and $\mathbf{H}$ are acoustic inertia and stiffness matrices, $\mathbf{q}$ is proportional to the external fluid displacement due to the motion of the stapes, $\rho_{f}$ is the fluid density and $\mathbf{R}$ denotes how the pressure is driven by the displacement of the plate elements, which is equal to $\mathbf{S}^{\mathrm{T}}$. For the coupled system these two equations can be combined to give

$$
\left[\begin{array}{cc}
\mathbf{M} & \mathbf{0} \\
\rho_{f} \mathbf{R} & \mathbf{Q}
\end{array}\right]\left[\begin{array}{c}
\ddot{\mathbf{w}} \\
\ddot{\mathbf{p}}
\end{array}\right]+\left[\begin{array}{cc}
\mathbf{K} & -\mathbf{S} \\
\mathbf{0} & \mathbf{H}
\end{array}\right]\left[\begin{array}{l}
\mathbf{w} \\
\mathbf{p}
\end{array}\right]=\left[\begin{array}{l}
\mathbf{0} \\
\mathbf{q}
\end{array}\right] .
$$

For single frequency excitation, proportional to $e^{i \omega t}$,

$$
\left[\begin{array}{cc}
\mathbf{K}-\omega^{2} \mathbf{M} & -\mathbf{S} \\
-\omega^{2} \rho_{f} \mathbf{R} & \mathbf{H}-\omega^{2} \mathbf{Q}
\end{array}\right]\left[\begin{array}{l}
\mathbf{w} \\
\mathbf{p}
\end{array}\right]=\left[\begin{array}{l}
\mathbf{0} \\
\mathbf{q}
\end{array}\right],
$$

where damping can now be incorporated by using complex elements in the stiffness matrix. This equation can also be written as a generalised dynamic stiffness matrix,

$$
\left[\begin{array}{ll}
\mathbf{D}_{11} & \mathbf{D}_{12} \\
\mathbf{D}_{21} & \mathbf{D}_{22}
\end{array}\right]\left[\begin{array}{l}
\mathbf{w} \\
\mathbf{p}
\end{array}\right]=\left[\begin{array}{l}
\mathbf{0} \\
\mathbf{q}
\end{array}\right]
$$

The solution of the full finite element method is obtained in the usual way by inversion of the dynamic stiffness matrix in Eq. (19), to give the vector of pressures and displacements at every node. The sparseness of the matrix can be used to develop efficient ways of inverting it, although these were not necessary for the relatively modest sized model used here. The modal BM velocity, defined in Eq. (1), can be obtained from a continuous BM displacement distribution, $w(x, y)$, using an equation analogous to Eq. (2) as

$$
v_{\mathrm{BM}}(x)=\frac{2 i \omega}{B} \int_{0}^{B} w(x, y) \sin \frac{\pi y}{B} d y .
$$

The finite element model provides the BM displacement in discrete form, as elements of the vector $\mathbf{w}$, which can be written in terms of the radial BM velocity distributions at each longitudinal slice along the cochlea as 


$$
\mathbf{w}=\left[\begin{array}{llll}
\mathbf{w}^{\mathrm{T}}(1) & \mathbf{w}^{\mathrm{T}}(2) & \cdots \mathbf{w}^{\mathrm{T}}(N)
\end{array}\right]^{\mathrm{T}},
$$

where $\mathbf{w}(n)$ is the radial BM displacement due to the plate motion at the $n$-th longitudinal element, and $N$ is the number of longitudinal elements, which is 512 in this case.

The modal BM velocity at the $n$-th position can then be estimated from the radial $\mathrm{BM}$ displacement distribution at this position using a discrete approximation to Eq. (20),

$$
v_{\mathrm{BM}}(n)=2 i \omega \mathbf{s}_{\mathrm{BM}}^{\mathrm{T}} \mathbf{w}(n),
$$

where $\mathbf{s}_{\mathrm{BM}}^{\mathrm{T}}$ is the vector of normalised values of the mode shape, $\sin (\pi y / B)$, at the nodal locations across the BM.

Figure 3 shows the distribution of modal BM velocity along the cochlea, calculated from the full finite element analysis, when driven by a tone of $1 \mathrm{kHz}$ at the stapes. Damping is included in the plate by introducing an imaginary component in each element of stiffness matrix, adjusted to give a damping ratio of 0.1 , as in Sec. II. The plate elements had a mass per unit area of $0.2 \mathrm{~kg} \cdot \mathrm{m}^{-2}$, which is rather larger than that used for the $3 \mathrm{D}$ fluid coupling case in Sec. II, as discussed below. Also shown in the figure is the modal BM velocity predicted using the WKB method from the wavenumber distribution of the slow wave calculated using the wave finite element analysis, as also discussed below.
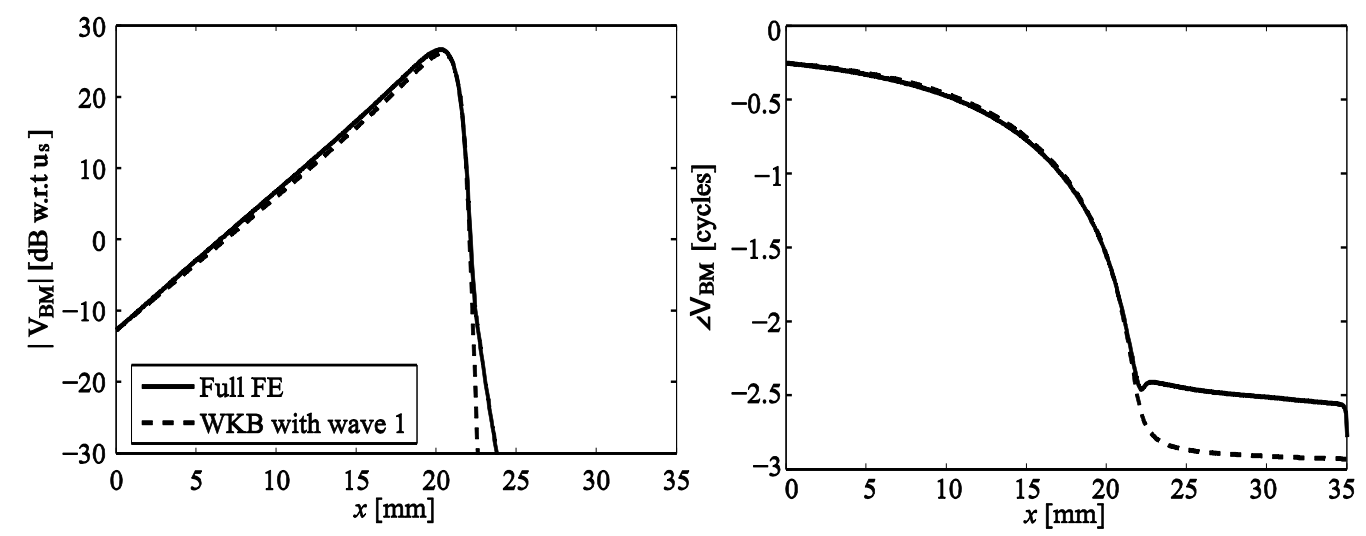

Fig. 3 The amplitude and phase of the modal BM velocity at $1 \mathrm{kHz}$, calculated from the full finite element model and the WKB method using the wavenumber distribution for the slow wave, wave 1, calculated using the wave finite element method. 


\section{b) Wave Finite Element}

In the present model of the cochlea, the $\mathrm{BM}$ is assumed to react only locally, so that longitudinal coupling only occurs via the pressure in the fluid chambers. The finite element of a single slice, or segment, at the $n$-th position along the cochlea, which in this case is a single element thick, can be written, following Eq. (19), as

$$
\left[\begin{array}{ll}
\mathbf{D}_{11}(n) & \mathbf{D}_{12}(n) \\
\mathbf{D}_{21}(n) & \mathbf{D}_{22}(n)
\end{array}\right]\left[\begin{array}{l}
\mathbf{w}(n) \\
\mathbf{p}(n)
\end{array}\right]=\left[\begin{array}{c}
\mathbf{0} \\
\mathbf{q}(n)
\end{array}\right]
$$

where $\mathbf{q}(n)$ is now the vector of fluid displacements at the interface of this segment of the FE model. This FE model can then be "condensed" by writing the first of the coupled equations in Eq. (23), as

$$
\mathbf{w}(n)=-\mathbf{D}_{11}^{-1}(n) \mathbf{D}_{12}(n) \mathbf{p}(n),
$$

so that the second coupled equation can be written as

$$
\left[\mathbf{D}_{22}(n)-\mathbf{D}_{21}(n) \mathbf{D}_{11}^{-1}(n) \mathbf{D}_{12}(n)\right] \mathbf{p}(n)=\mathbf{q}(n),
$$

where the term in square brackets can be written as a single condensed dynamic stiffness matrix $\tilde{\mathbf{D}}(n)$. A similar method can be used to condense any internal degrees of freedom within the segment if it is more than one element thick (Mace et al., 2005; Duhamel et al., 2006).

If the vector of fluid displacements and the vector of pressures are now partitioned into components of the left and right hand side of the segment, as shown in Fig. 4, so that

$$
\mathbf{p}(n)=\left[\begin{array}{l}
\mathbf{p}_{\mathrm{L}}(n) \\
\mathbf{p}_{\mathrm{R}}(n)
\end{array}\right], \mathbf{q}(n)=\left[\begin{array}{l}
\mathbf{q}_{\mathrm{L}}(n) \\
\mathbf{q}_{\mathrm{R}}(n)
\end{array}\right],
$$

then we can write

$$
\left[\begin{array}{cc}
\tilde{\mathbf{D}}_{\mathrm{LL}}(n) & \tilde{\mathbf{D}}_{\mathrm{LR}}(n) \\
\tilde{\mathbf{D}}_{\mathrm{RL}}(n) & \tilde{\mathbf{D}}_{\mathrm{RR}}(n)
\end{array}\right]\left[\begin{array}{l}
\mathbf{p}_{\mathrm{L}}(n) \\
\mathbf{p}_{\mathrm{R}}(n)
\end{array}\right]=\left[\begin{array}{c}
\mathbf{q}_{\mathrm{L}}(n) \\
\mathbf{q}_{\mathrm{R}}(n)
\end{array}\right] .
$$


where $\tilde{\mathbf{D}}_{\mathrm{LL}}, \tilde{\mathbf{D}}_{\mathrm{LR}}, \tilde{\mathbf{D}}_{\mathrm{RL}}$ and $\tilde{\mathbf{D}}_{\mathrm{RR}}$ are the partitioned sub-matrices of the condensed dynamic stiffness matrix $\tilde{\mathbf{D}}$. The terms of this equation can be re-arranged to express the fluid displacements and pressures on one side of the $n$-th segment in terms of those on the other side, so that

$$
\left[\begin{array}{c}
\mathbf{p}_{\mathrm{R}}(n) \\
-\mathbf{q}_{\mathrm{R}}(n)
\end{array}\right]=\mathbf{T}(n)\left[\begin{array}{c}
\mathbf{p}_{\mathrm{L}}(n) \\
\mathbf{q}_{\mathrm{L}}(n)
\end{array}\right],
$$

where the transfer matrix for the $n$-th segment is equal to

$$
\mathbf{T}(n)=\left[\begin{array}{cc}
-\tilde{\mathbf{D}}_{\mathrm{LR}}^{-1}(n) \tilde{\mathbf{D}}_{\mathrm{LL}}(n) & \tilde{\mathbf{D}}_{\mathrm{LR}}^{-1}(n) \\
-\tilde{\mathbf{D}}_{\mathrm{RL}}(n)+\tilde{\mathbf{D}}_{\mathrm{RR}}(n) \tilde{\mathbf{D}}_{\mathrm{LR}}^{-1}(n) \tilde{\mathbf{D}}_{\mathrm{LL}}(n) & -\tilde{\mathbf{D}}_{\mathrm{RR}}(n) \tilde{\mathbf{D}}_{\mathrm{LR}}^{-1}(n)
\end{array}\right]
$$

We now assume that a particular distribution of pressures and volume velocities, due to the $m$-th mode, on the right hand side of the segment is equal to that on the left hand side of the segment, apart from a complex constant of proportionality $\lambda_{m}$, so that

$$
\left[\begin{array}{c}
\mathbf{p}_{\mathrm{R}}(n, m) \\
-\mathbf{q}_{\mathrm{R}}(n, m)
\end{array}\right]=\lambda_{m}\left[\begin{array}{c}
\mathbf{p}_{\mathrm{L}}(n, m) \\
\mathbf{q}_{\mathrm{L}}(n, m)
\end{array}\right]
$$

This distribution would thus propagate as a wave with an unaltered shape along a uniform cochlea, with a wavenumber, $k_{m}$, determined by

$$
\lambda_{m}=e^{-i k_{m} \Delta},
$$

where $\Delta$ is the length of the segment. The right hand side of Eq. (31) must now be equal to the right hand side of Eq. (29), and so $\lambda_{m}$, and the corresponding distribution of pressures and fluid displacements, must be an eigenvalue, and the corresponding eigenvector, of the transfer matrix for this segment. 


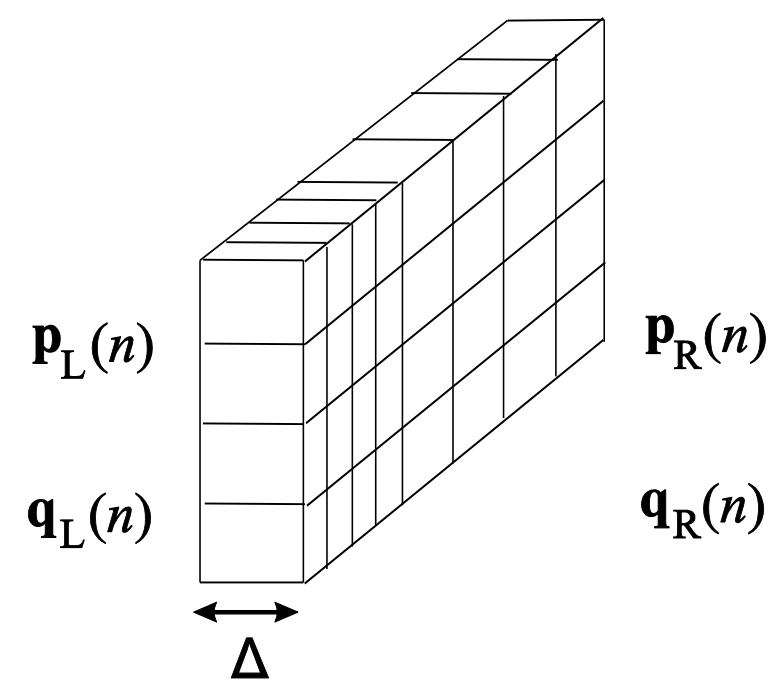

Fig. 4 An elemental segment of the cochlea used in the wave finite element method showing the pressure and displacement on the left hand and right hand side of this element. The internal structure of the cochlea is not shown.

Using the wave finite element method, the wavenumbers are thus obtained directly from the eigenvalues of the transfer matrix for the 3D finite element model of the segment, rather than eigenvalue problem for a finite element model of a $2 \mathrm{D}$ slice of the cochlea being used to deduce a polynomial dispersion equation, which then has to be solved to give the wavenumber (Fuhrmann et al., 1987; Chadwick et al., 1996; Steele, 1999). The analysis of a 2D slice is called the semi-analytic finite element method in the engineering literature, which dates back to the 1970's, as discussed by Bartoli et al. (2006) for example, or the spectral finite element method (Finnveden, 2004), and has also been extended to deal with fluidstructural problems, by Nilsson and Finnveden (2008) for example. An advantage of the wave finite element method over the semi-analytic finite element method is that the same 3D finite element models used to calculate the properties of the segments can also be joined together to form the full FE of the whole cochlea, as in Sec. III (a). It is also possible to analyse segments of finite thickness using the wave finite element. In the context of cochlear mechanics, each segment could incorporate finite element models of several layers of hair 
cells. It would be also possible to incorporate asymmetries into the structure, by slanting the hair cells in the longitudinal direction, for example.

The wave finite element analysis becomes a little more complicated than for uniform structures when the structural parameters of the cochlea vary along its length, as considered by $\mathrm{Ni}$ et al. (2010). In this case the eigenvector corresponding to a specific type of wave is not exactly the same when passing from one segment to the next. If the longitudinal variation in parameters is gradual, however, the change in the mode shape corresponding to this eigenvector will not be very large from one element to the next. By calculating the inner products of the left eigenvectors for one element with the right eigenvector for the adjacent element $(\mathrm{Ni}, 2012)$, it is then possible to track which eigenvalue, and hence which wavenumber, is associated with each mode travelling along the cochlea. Figure 5 (a) and (b), for example, shows the variation with longitudinal position of the real and imaginary parts of the wavenumber associated with some of the different waves that can propagate along the discrete cochlear model at $1 \mathrm{kHz}$. These waves have negative imaginary wavenumbers and are thus forward travelling waves. The first five have been chosen as the waves with the least-negative imaginary component of the wavenumber and the fifth wave has been selected because its radial BM motion corresponds to a higher order bending mode. The BM velocity distribution associated with each of these waves, close to the position with the largest real component of the wavenumber, is plotted in Fig. 5 (c). The associated pressure distributions in the two fluid chambers at $x=20 \mathrm{~mm}$ are plotted for these waves in Fig. 6 . 

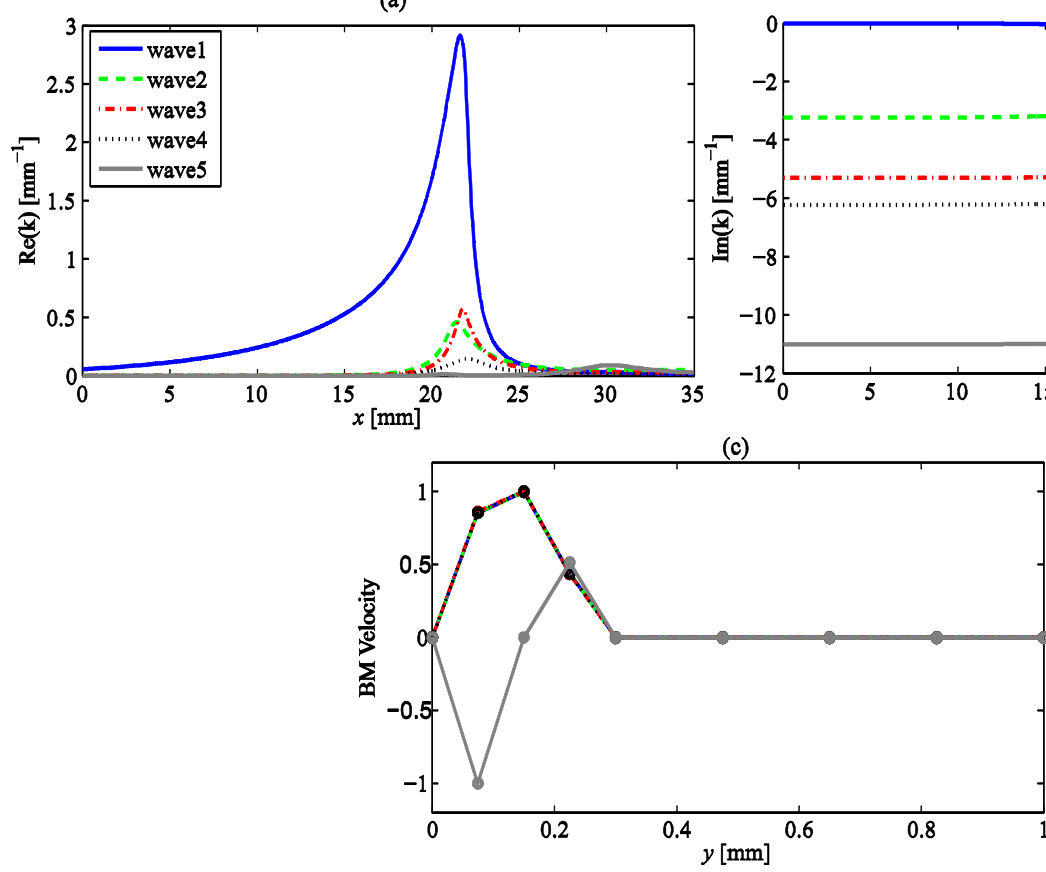
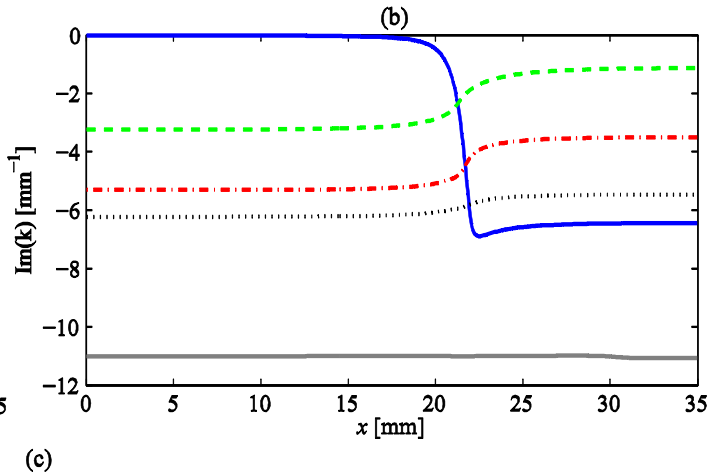

Fig. 5 (a-b) Wave number distribution of 5 of the forward going waves, calculated using the wave finite element model of the cochlea at $1 \mathrm{kHz}$. Also shown, (c) is the normalised BM velocity in the radial direction associated with the 5 selected waves, calculated at the place where the real part of their wavenumber is largest (colour online).
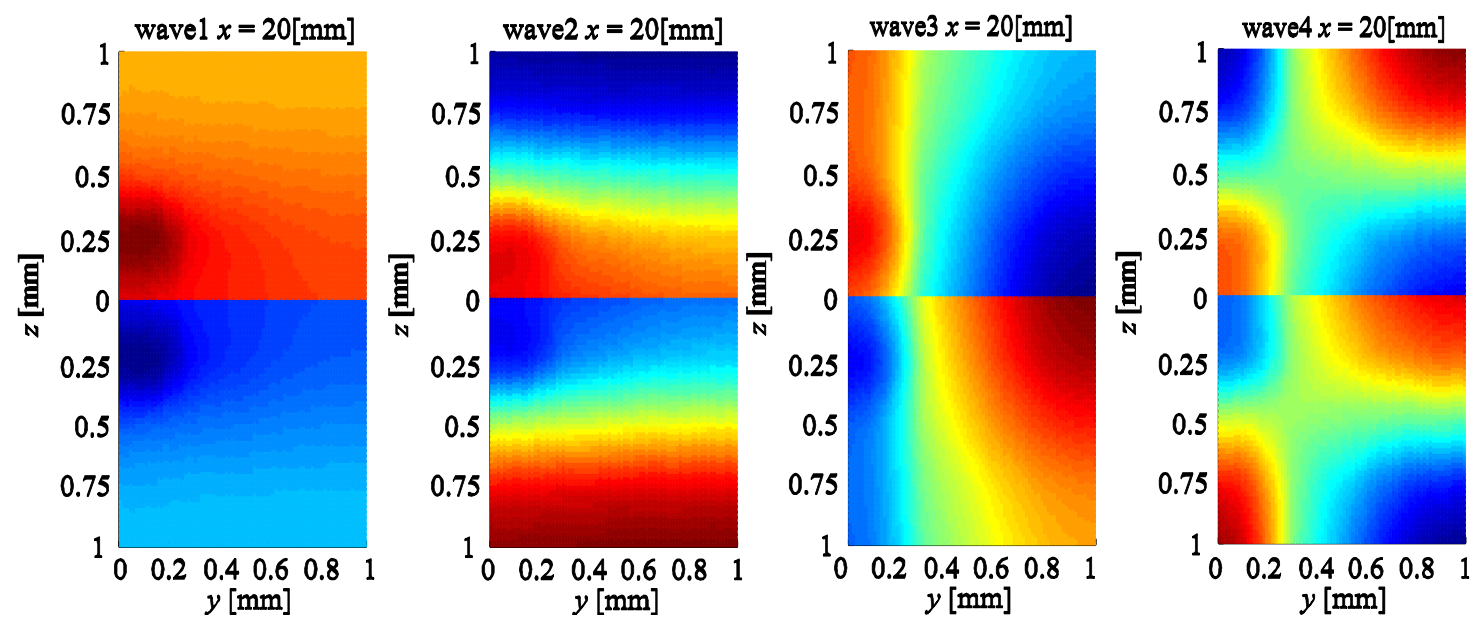

The finite element model for each of the 512 segments of the cochlea has $8 \times 4$ hexahedral elements to describe the fluid motion in each chamber, and 4 quadrilateral elements along the BM to describe its radial structural response as a beam. There are thus $9 \times 5$ nodes on each face of the fluid chamber slice, each having 1 degree of freedom, and $5 \times 1$ nodes on each edge of the BM slice, each having 3 degrees of freedom. The BM elements are assumed to be 
separated from each other in the longitudinal direction, however, so that the degrees of freedom associated with the $\mathrm{BM}$ elements are all condensed, as described above, and the vectors in Eq. (29), for example, have 90 degrees of freedom. There are thus 90 eigenvalues of the transfer matrix $\mathbf{T}$ in Eq. (29) and hence the wavenumbers of 90 separate waves can be calculated. Only half of these will be propagating in the forward direction, however, and most of these have wavenumbers with large imaginary components and thus are heavily attenuated, even a short distance from the excitation position. In fact only the wave labelled 1 in Fig. 5 (a) has a small imaginary part to its wavenumber at the base, and is thus able to propagate any significant distance along the cochlea.

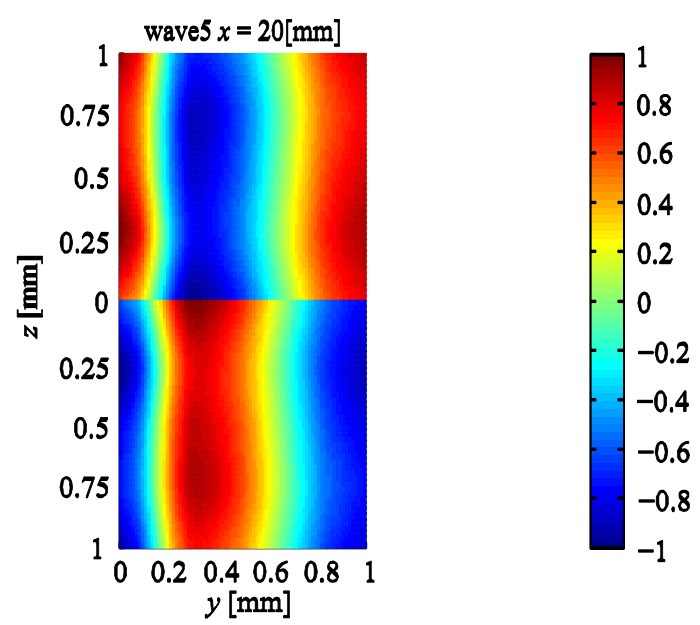

Fig. 6 The pressure distribution in the fluid chambers associated with each of the selected waves at $x=20 \mathrm{~mm}$, normalised with respect to their maximum value. Note that an interpolation has been used to visualize the pressure distribution, which are only calculated for the $8 \times 4$ elements shown in Fig. 4. Only the distribution in one chamber has been calculated, since symmetry is assumed, the pressure distribution in the other chamber, which is the same with that calculated but with opposite sign, is also plotted for purpose of illustration.

This wave has a radial BM velocity distribution that is similar to the first bending mode and a pressure distribution which is in phase across each fluid chamber, but of opposite sign in the upper and lower chambers. It thus corresponds to the conventional "slow wave" in cochlea mechanics, where the stiffness of the BM interacts with the inertia of the fluid in the chambers. The pressure distributions shown in Fig. 6 for waves 2 to 4 correspond to higher- 
order fluid modes, whose pressure distributions have both positive and negative components in each chamber. These waves are evanescent, since they have a significantly negative imaginary component in their wavenumbers. Wave 5 is due to the second bending mode of the BM, which cuts-on at more apical positions along the cochlea and has a positive and negative distribution along the face of the BM in Fig. 6. A wave not shown, which has a very small real wavenumber and no significant BM velocity, corresponds to the fast wave due to acoustic propagation within the fluid. This is present if both fluid chambers are modelled and the finite element model of the fluid is assumed to have a finite compressibility.

One difference between the wavenumber distribution for wave 1 calculated using the WFE method, in Fig. 5, from that calculated analytically, in Fig. 2, is that the imaginary part of the wavenumber at apical positions is much more negative than for wave 2 . This behaviour was found to be dependent on the BM damping, such that if the effective damping ratio were greater than about 0.14 in this WFE model, the imaginary part of wave 1 had a less negative imaginary part than wave 2 at apical locations, as observed in the analytic result. In fact a similar change in behaviour with damping can be seen in the polar plots of wavenumber shown in Fig. 4 of Watts (Watts, 2000), where the "cut-off mode" has a more negative imaginary component than the "travelling wave mode" at $400 \mathrm{~Hz}$, where the BM is heavily damped, but the opposite is shown at $800 \mathrm{~Hz}$, where the BM is less heavily damped.

It is not strictly necessary in the passive case to use as many longitudinal elements in the finite element model as used above. The minimum wavelength of the slow wave is about 2.2 $\mathrm{mm}$, since the peak real wavenumber is about $2.9 \mathrm{~mm}^{-1}$, and so there are at least 32 elements per wavelength. Simulations have also been performed with 256 longitudinal elements and 8 by 4 elements in each cross section, which gives results that are very similar to those shown here. There are, however, still potential numerical errors for waves with very large imaginary wavenumbers, since the decay length is then small compared to the size of an element (Waki 
et al., 2009b). Since these waves make a negligible contribution to the full finite element results, however, such errors are not particularly significant in this application. The details of the wavenumber distributions in Fig. 5 are, however, dependent on the number of fluid elements used in the cross section. In order to correctly reproduce the near field fluid pressure very close to the BM a much denser grid of fluid elements would be required than has been used here. The only important property of this near field pressure for wave 1 , however, is the added mass of the fluid. This is somewhat smaller for the FE grid used here than it was for the analytic model in Sec. II, so that the BM mass was increased to account for this effect, as mentioned above. Waves 2 to 4 correspond to higher order fluid modes, which have relatively simple cross-sectional mode shapes that can be accurately reproduced with the current grid density in the FE model. The most important aspect of these waves is the value of the negative part of the wavenumber in the basal region, and this is not significantly affected if the number of fluid elements is increased. The relatively coarse grid of fluid elements used here thus correctly predicts the important features of the wavenumbers in the WFE analysis. Assuming that the slow wave propagates in isolation, the WKB method can be used with the wavenumber distribution of wave 1 in Fig. 5 (a), to calculate a longitudinal distribution of the BM velocity, whose magnitude and phase is shown in Fig. 3, along with the results of the full finite element method. The results of the full finite element model are thus in reasonable agreement with those predicted using only the slow wave up to about $x=22 \mathrm{~mm}$. The less rapid fall off in the results of the full finite element model just apical of the peak, compared those using the WKB method, has also been noted by Steele and Taber (1979), de Boer and Viergever (1982) and Watts (2000), as discussed above. 


\section{Decomposition into wave components}

We now consider a more systematic decomposition of the results of the full finite element analysis into wave components. We assume that the $N \times N$ transfer matrix has a linearly independent set of $N$ eigenvectors, and express the eigenvalue, eigenvector decomposition of the transfer matrix for the $n$-th segment in the form

$$
\mathbf{T}(n)=\mathbf{Q}(n) \mathbf{\Lambda}(n) \mathbf{Q}^{-1}(n),
$$

where $\Lambda(n)$ is the diagonal matrix of eigenvalues, the right eigenvectors of $\mathbf{T}(n)$ correspond to the columns of $\mathbf{Q}(n)$ and the left eigenvectors of $\mathbf{T}(n)$ correspond to the rows of $\mathbf{Q}^{-1}(n)$. Using Eq. (33), Eq. (29) can also be written as

$$
\mathbf{Q}^{-1}(n)\left[\begin{array}{c}
\mathbf{p}_{\mathrm{R}}(n) \\
-\mathbf{q}_{\mathrm{R}}(n)
\end{array}\right]=\boldsymbol{\Lambda}(n) \mathbf{Q}^{-1}(n)\left[\begin{array}{c}
\mathbf{p}_{\mathrm{L}}(n) \\
\mathbf{q}_{\mathrm{L}}(n)
\end{array}\right]
$$

Since $\boldsymbol{\Lambda}(n)$ is diagonal, the inner product of each row of $\mathbf{Q}^{-1}(n)$ with the state vectors on the right and left hand side gives an uncoupled set of equations of the form

$$
a_{\mathrm{R}}(n, m)=\lambda_{m}(n) a_{\mathrm{L}}(n, m),
$$

where $a_{\mathrm{R}}(n, m)$ and $a_{\mathrm{L}}(n, m)$ can be interpreted as the complex amplitudes of the $m$-th wave on the right and left hand side of the $n$-th segment (Duhamel et al., 2006). The vector of all such wave amplitudes on the right hand side of this segment can be written as

$$
\mathbf{a}_{\mathrm{R}}(n)=\mathbf{Q}^{-1}(n)\left[\begin{array}{c}
\mathbf{p}_{\mathrm{R}}(n) \\
-\mathbf{q}_{\mathrm{R}}(n)
\end{array}\right],
$$

where the fluid displacements can be calculated from the pressures using Eq. (25), and so the wave amplitudes in the $n$-th segment can be expressed entirely as a function of the vector of elemental pressures on the face of this segment, calculated from the full finite element model. 
The state vector due to all of the wave amplitudes can be calculated by multiplying both sides of Eq. (36) by $\mathbf{Q}(n)$, so that the contribution to the state vector due to the $m$-th wave on the right hand side of the $n$-th segment can be defined as

$$
\left[\begin{array}{c}
\mathbf{p}_{\mathrm{R}}(n, m) \\
-\mathbf{q}_{\mathrm{R}}(n, m)
\end{array}\right]=\mathbf{r}(n, m) a_{\mathrm{R}}(n, m),
$$

where $\mathbf{r}(n, m)$ is the $m$-th column vector of $\mathbf{Q}(n)$ and $a_{\mathrm{R}}(n, m)$ is one element of $\mathbf{a}_{\mathrm{R}}(n)$ in Eq. (36).

The vector of elemental $\mathrm{BM}$ displacements due to the $m$-th wave, $\mathbf{w}_{\mathrm{R}}(n, m)$, can then be calculated from the vector of elemental pressures using Eq. (24), from which the modal BM velocity due to the $m$-th wave on the right hand side of the $n$-th segment, $v_{\text {Вм }}(n, m)$, can be calculated using a modified form of Eq. (22) as

$$
v_{\mathrm{BM}}(n, m)=2 i \omega \mathbf{s}_{\mathrm{BM}}^{\mathrm{T}} \mathbf{w}_{\mathrm{R}}(n, m),
$$

where $\mathbf{s}_{\mathrm{BM}}^{\mathrm{T}}$ is the vector of normalised values of the BM mode shape used in Eq. (22).

The contributions to the overall modal BM velocity distributions, due to each of the forward going waves selected in Fig. 5, are plotted in Fig. 7. The WKB result for wave 1 is seen to be in reasonable agreement with the calculated contribution of this wave to the full finite element results. The contribution of wave 1 , however, is significantly less than the overall result of the full finite element solution for positions beyond the peak response at this frequency, at about $22 \mathrm{~mm}$ along the cochlea, where the contribution of wave 2 begins to dominate the overall response. The contribution of wave 2, which is an evanescent higherorder fluid mode, decays away on either side of this peak, as do the contributions of wave 3 , 4 and 5, although the amplitudes of these waves are too small to significantly affect the overall response. 

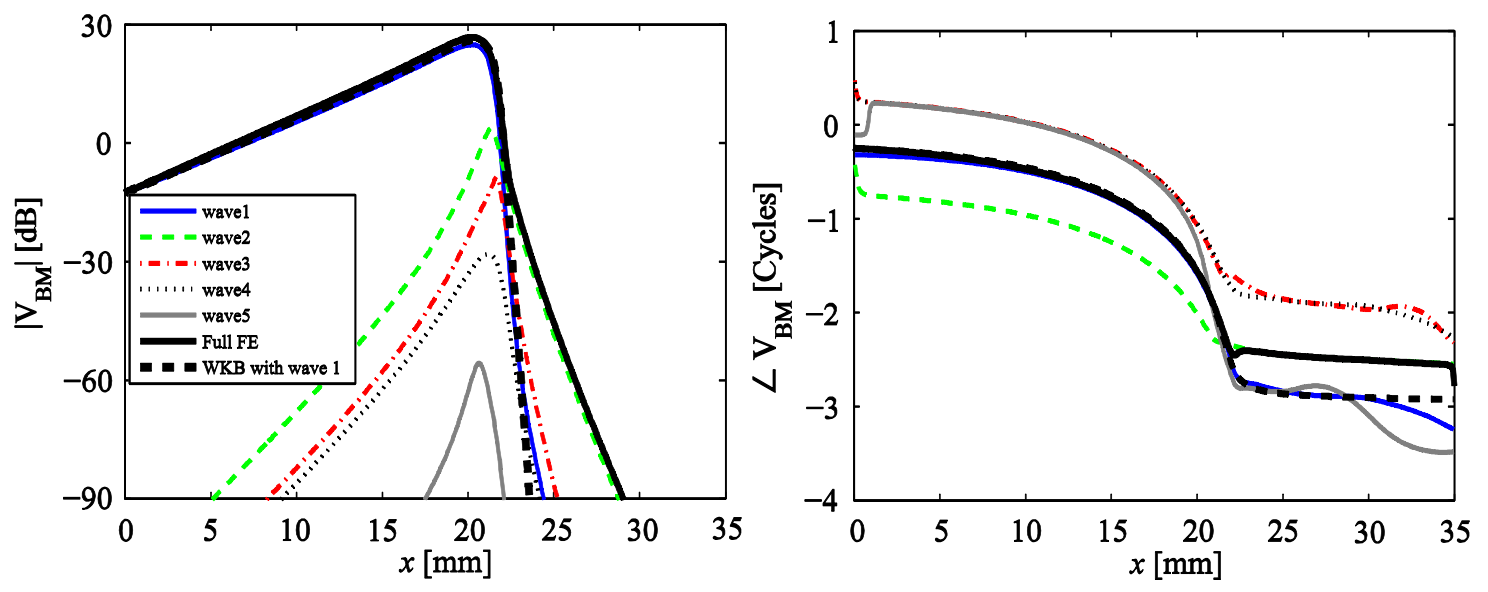

Fig. 7 Decomposition of the modal BM velocity into components due to each of the forward travelling waves in Fig. 5, the WKB reconstruction of the slow wave and the modal BM velocity from the full finite element model are also shown (colour online).

In principle the contributions of the backwards travelling waves can also be calculated using Eq. (37), using the columns of the eigenvector matrix corresponding to these waves. The amplitude of the backwards travelling slow wave, calculated using this method, is, however, only $10 \mathrm{~dB}$ to $20 \mathrm{~dB}$ below that of the forward travelling wave, and its phase, tellingly, is also very similar to that of the forward travelling wave. It is believed that this estimate of the backwards travelling wave component is significantly in error, however, because of the inherent assumption in the wave finite element method that the waves are propagating in a uniform system. The wave impedances, relating the pressure difference and longitudinal fluid velocity in the fluid ducts, are the same for both forward and backward going waves in such a uniform system, which is reflected in the similarity of the eigenvectors of the transfer matrix in Eq. (29), apart from the sign change associated with the direction of the fluid velocity (Mace et al., 2005).

In fact if a WKB solution is taken for the pressure and fluid velocity of the slow wave in a 1D but non-uniform system, the ratio of these two quantities is given by

$$
\left[\frac{p(x)}{u(x)}\right]_{ \pm}=2 \rho c\left(1 \mp \frac{i}{2 k^{2}} \frac{\partial k}{\partial x}\right)^{-1}
$$


where the subscript + or - denotes the solution for forward and backward going waves. The current WFE method does not take the $\frac{i}{2 k^{2}} \frac{\partial k}{\partial x}$ term into account in the mode shape, since it assumes the system is uniform, and this generates spurious backward going components, even if only a forward going wave is, in fact, present.

The WKB solution, however, assumes that, for a given wave with wavenumber $k_{m}(n)$, the vector of pressures, $\mathbf{p}(n)$, varies as $\left[k_{m}(n) \Delta\right]^{-1 / 2} \mathbf{c}_{m}(n)$ and the vector of fluid velocity, $\mathbf{q}(n)$, varies as $\left[k_{m}(n) \Delta\right]^{1 / 2} \mathbf{d}_{m}(n)$, where the elemental thickness, $\Delta$, is introduced to normalise the wavenumbers and $\mathbf{c}_{m}(n)$ and $\mathbf{d}_{m}(n)$ are the WKB components that vary only in phase. It is possible to re-formulate the transfer matrix in Eq. (29) so that it relates the components of $\mathbf{c}_{m}(n)$ and $\mathbf{d}_{m}(n)$ on the left and right hand side of the elements. The eigenvalues of this matrix are then equal to $e^{-i k_{m} \Delta}$, even if the system is not uniform. This new transfer matrix must be calculated for each individual mode using an iterative procedure, however, since it depends on the wavenumber distribution, $k_{m}(n)$, which can, for example, be estimated using the normal WFE method. In practice the wavenumber distributions calculated from the eigenvalues of the transfer matrix for $\mathbf{c}(n)$ and $\mathbf{d}(n)$ are not significantly different from those calculated from the transfer matrix for $\mathbf{p}(n)$ and $\mathbf{q}(n)$. There is, however, a significant difference in the eigenvector structure of these two transfer matrices, which determines the mode shapes used to calculate the modal amplitudes in Eq. (37). The changes to these new eigenvectors hardly affect the estimated amplitudes of the forward going waves but significantly reduce the amplitude of the backward going wave, so that its amplitude are now about $100 \mathrm{~dB}$ less than that of the forward going waves and are thus negligible. The details of this new formulation are still under investigation. 


\section{Discussion and Conclusions}

Our understanding of cochlea mechanics is largely based, either explicitly or implicitly, on the assumption that only a single type of "slow wave" propagates along its length. Some of the properties of this wave can be calculated from a simple model of the passive cochlea that includes a locally-reacting BM and 1D fluid coupling. A useful description of such a wave, at a given frequency, is the distribution of its complex wavenumber along the length of the cochlea. The real part of this wavenumber describes the change of phase with distance and determines the wave speed. The imaginary part of the wavenumber describes the change of amplitude with distance and must be negative for a forward travelling wave in the passive cochlea, since energy can only be dissipated. Assuming that the wavenumber does not change too rapidly with position, and that the wave travels without interference from other waves, the coupled response of the cochlea can be deduced from the wavenumber distribution using the WKB method.

In general, however, there are many other mechanisms, apart from 1D fluid coupling, that give rise to longitudinal coupling in the cochlea, particularly the higher order modes associated with 3D fluid motion. A simple model of wave dispersion that includes such effects is considered, in order to calculate the wavenumber distributions of the additional waves that they generate. The wavenumber distribution derived from an approximation to 3D fluid coupling shows that, in the basal region, the additional wave in the coupled system has similar characteristics to a cut off acoustic mode, since the wavenumber is largely imaginary. Even though these additional waves may exist, it is not clear what role they play in normal passive cochlear function, particularly the extent to which they are excited when the cochlea is driven normally, via the stapes from the middle ear.

The fully coupled response of the cochlea to middle ear excitation can be calculated using a numerical model, such as that obtained using the finite element method, although the insight 
gained from the wave approach is then lost. The wave finite element, WFE, method is used here to predict the properties of as many types of waves as there are degrees of freedom across each cross section of the finite element model. Almost all of the forward travelling components of these waves have large negative imaginary components, indicating that they decay away very quickly. Examples of such evanescent waves include the higher order fluid modes and also modes associated with more complicated radial distributions of BM motion than is associated with the slow wave.

The mode shapes associated with the waves predicted from the WFE analysis are then used to decompose the results of the full finite element model into wave components. In a uniform system there would be no coupling between the modes, which would propagate independently. Due to the variation of parameters along the cochlea, however, the wavenumbers are functions of longitudinal position, and in such non-homogeneous systems one wave is able to scatter into other types of waves. In the passive cochlea model used here, with a locally reacting $\mathrm{BM}$, this is only seen to occur to a significant extent in the region where the slow wave is rapidly decaying, as previously considered by Watts (Watts, 2000).

The framework provided by the WFE method also allows the analysis of more complicated models of the cochlear mechanics. If the BM dynamics were active, but still locally reacting, and the same approximation to the 3D fluid coupling were used as given by Eq. (8), the dispersion equation for the coupled response would still be given by Eq. (9). Although the properties of the slow wave will be modified by the active components of $Z_{\mathrm{BM}}$ in this case, the other wave would still largely be determined by the evanescent higher order fluid mode. It is only when additional forms of longitudinal coupling are included in the model, that multiple propagating modes might be expected. There are a number of mechanisms for longitudinal coupling along the organ of Corti and BM and it is unclear how these might behave together, or interact with multiple fluid chambers, to determine the types of wave that 
can propagate. These mechanisms include orthotropy in the BM (Meaud and Grosh, 2010), tectorial membrane elasticity (Zwislocki and Kletsky, 1979; Ghaffari et al., 2007) longitudinal electrical coupling between the hair cells (Ramamoorthy et al., 2007) and the feedforward action of the outer hair cells (Steele et al., 1993; de Boer, 1996).

Finite element models have been used to investigate the effects of several of these longitudinal coupling mechanisms on the overall BM response (Cai et al., 2004; Parthasarathi et al., 2000; Baumgart et al., 2008). The wave finite element method provides the opportunity to analyse the types of wave that can propagate in such models and, more importantly, decompose the response of fully-coupled finite element models into the components due to each of these waves, in order to study how they interact. In this way the insight provided by the wave approach can be brought to bear on numerical models that incorporate various detailed features of the cochlea, and allow us to analyse their significance in the overall response.

\section{ACKNOWLEDGMENT}

This work was partly supported by EPSRC Grant No. EP/K003836/1.

Bartoli, I., Marzani, A., Lanza di Scalea, F., and Viola, E. (2006). "Modeling wave propagation in damped waveguides of arbitrary crosssection,” J. Sound Vib. 295(3-5), 685-707.

Baumgart, J., Chiaradia, C., Fleischer, M., Yarin, Y., Grundmann, R., and Gummer, A. W. (2008). "Fluid mechanics in the subtectorial space," in Proceedings of the 10th International Workshop on the Mechanics of Hearing, Keele University, Staffordshire, UK, pp. 288-293.

Cai, H., and Chadwick, R. (2003). "Radial structure of traveling waves in the inner ear," SIAM J. Appl. Math 63(4), 1105-1120.

Cai, H., Shoelson, B., and Chadwick, R. S. (2004). "Evidence of tectorial membrane radial motion in a propagating mode of a complex cochlear model,” Proc. Natl. Acad. Sci. U.S.A. 101(16), 6243-6248.

Chadwick, R. S., Dimitriadis, E. K., and Iwasa, K. H. (1996). "Active control of waves in a cochlear model with subpartitions," Proc. Natl. Acad. Sci. U.S.A. 93(6), 2564-2569.

de Boer, E. (1996). "Mechanics of the cochlea: Modelling efforts," in The Cochlea, edited by P. Dallos, A. N. Popper, and R. R. Fay (Springer, New York), pp. 258-317.

de Boer, E. (1998). “A method for forward and inverse solutions of a three-dimensional model of the cochlea," J. Acoust. Soc. Am. 103(6), 3725-3728.

de Boer, E., and Mackay, R. (1980). “Reflections on reflections,” J. Acoust. Soc. Am. 67(3), 882-890. 
de Boer, E., and Viergever, M. A. (1982). "Validity of the Liouville-Green (or WKB) method for cochlear mechanics," Hear. Res. 8(2), 131-155.

Duhamel, D., Mace, B. R., and Brennan, M. J. (2006). "Finite element analysis of the vibrations of waveguides and periodic structures," J. Sound Vib. 294(1-2), 205-220.

Elliott, S. J., Lineton, B., and Ni, G. (2011). "Fluid coupling in a discrete model of cochlear mechanics," J. Acoust. Soc. Am. 130(3), 1441-1451.

Fahy, F., and Gardonio, P. (2007). Sound and Structural Vibration: Radiation, Transmission and Response (Academic Press, Oxford, UK), pp. 500.

Finnveden, S. (2004). "Evaluation of modal density and group velocity by a finite element method," J. Sound Vib. 273(1-2), 51-75.

Fuhrmann, E., Schneider, W., and Schultz, M. (1987). "Wave propagation in the cochlea (inner ear): Effects of Reissner's membrane and nonrectangular cross-section," Acta Mech. 70(1-4), 15-30.

Ghaffari, R., Aranyosi, A. J., and Freeman, D. M. (2007). "Longitudinally propagating traveling waves of the mammalian tectorial membrane,” Proc. Natl. Acad. Sci. U.S.A. 104(42), 16510-16515.

Lamb, J. S., and Chadwick, R. S. (2011). "Dual traveling waves in an inner ear model with two degrees of freedom," Phys. Rev. Lett. 107(8), 088101.

Mace, B. R., Duhamel, D., Brennan, M. J., and Hinke, L. (2005). "Finite element prediction of wave motion in structural waveguides,” J. Acoust. Soc. Am. 117(5), 2835-2843.

Meaud, J., and Grosh, K. (2010). "The effect of tectorial membrane and basilar membrane longitudinal coupling in cochlear mechanics," J. Acoust. Soc. Am. 127(3), 1411-1421.

Ni, G. (2012). "Fluid coupling and waves in the cochlea," Ph.D., University of Southampton, Southampton, pp. $87-89$.

Ni, G., Elliott, S. J., and Mace, B. R. (2010). “A fluid-structural model of the cochlea using wave finite element method," in Proceedings of the Tenth International Conference on Recent Advances in Structural Dynamics (RASD2010), Southampton, UK, pp. 12-14.

Nilsson, C. M., and Finnveden, S. (2008). "Waves in thin-walled fluid-filled ducts with arbitrary cross-sections," J. Sound Vib. 310(1-2), 58-76.

Ramamoorthy, S., Deo, N. V., and Grosh, K. (2007). "A mechano-electroacoustical model for the cochlea: Response to acoustic stimuli,” J. Acoust. Soc. Am. 121(5), 2758-2773.

Shera, C. A. (2007). "Laser amplification with a twist: Traveling-wave propagation and gain functions from throughout the cochlea,” J. Acoust. Soc. Am. 122(5), 2738-2758.

Steele, C. R. (1999). “Toward three-dimensional analysis of cochlear structure,” ORL 61(5), 238-251.

Steele, C. R., Baker, G., Tolomeo, J., and Zetes, D. (1993). "Electromechanical models of the outer hair cell," in Biophysics of Hair-Cell Sensory Systems, edited by H. Duifhuis, J. W. Horst, P. v. Dijk, and S. M. V. Netten (World Scientific, Singapore), pp. 207-214

Steele, C. R., and Taber, L. A. (1979a). "Comparison of WKB and finite difference calculations for a twodimensional cochlear model,” J. Acoust. Soc. Am. 65(4), 1001-1006.

Steele, C. R., and Taber, L. A. (1979b). "Comparison of WKB calculations and experimental results for threedimensional cochlear models,” J. Acoust. Soc. Am. 65(4), 1007-1018. 
Taber, L. A., and Steele, C. R. (1981). "Cochlear model including three-dimensional fluid and four modes of partition flexibility,” J. Acoust. Soc. Am, 70(2), 426-436.

Thompson, D. J. (1993). “Wheel-rail noise generation, part 3: Rail vibration,” J. Sound Vib. 161(3), 421-446.

Waki, Y., Mace, B. R., and Brennan, M. J. (2009a). "Free and forced vibrations of a tire using a wave/finite element approach,” J. Sound Vib. 323(3-5), 737-756.

Waki, Y., Mace, B. R., and Brennan, M. J. (2009b). "Numerical issues concerning the wave and finite element method for free and forced vibrations of waveguides," J. Sound Vib. 327(1-2), 92-108.

Watts, L. (2000). "The mode-coupling Liouville-Green approximation for a two-dimensional cochlear model," J. Acoust. Soc. Am. 108(5), 2266-2271.

Zweig, G., Lipes, R., and Pierce, J. R. (1976). “The cochlear compromise,” J. Acoust. Soc. Am. 59(4), 975-982. Zwislocki, J. J. (1950). “Theory of the acoustical action of the cochlea,” J. Acoust. Soc. Am. 22(6), 778-784.

Zwislocki, J. J., and Kletsky, E. J. (1979). "Tectorial membrane: A possible effect on frequency analysis in the cochlea," Science 204(4393), 639-641. 